\title{
Efficacy of fecal microbiota therapy in steroid dependent ulcerative colitis: a real world intention-to-treat analysis
}

\author{
Ajit Sood ${ }^{1}$, Ramit Mahajan ${ }^{1}$, Garima Juyal ${ }^{2}$, Vandana Midha ${ }^{3}$, Charanpreet Singh Grewal $^{1}$, Varun Mehta $^{1}$, \\ Arshdeep Singh ${ }^{1}$, Mohan C Joshi ${ }^{4}$, Vikram Narang ${ }^{5}$, Kirandeep Kaur ${ }^{6}$, Hasrat Sidhu ${ }^{3}$ \\ ${ }^{1}$ Department of Gastroenterology, Dayanand Medical College \& Hospital, Ludhiana; ${ }^{2}$ School of Biotechnology, Jawaharlal Nehru University, \\ New Delhi; ${ }^{3}$ Department of Internal Medicine, Dayanand Medical College \& Hospital, Ludhiana; ${ }^{4}$ Multidisciplinary Centre for Advanced \\ Research and Studies (MCARS), Jamia Millia Islamia, New Delhi; Departments of ${ }^{5}$ Pathology and ${ }^{6}$ Pharmacology, Dayanand Medical College \\ \& Hospital, Ludhiana, India
}

Background/Aims: Four high-quality randomized controlled trials have proven the efficacy of fecal microbiota transplantation (FMT) in active ulcerative colitis (UC). We assessed the efficacy of FMT in a real-world setting involving steroid-dependent patients with UC. Methods: This was a single-center prospective analysis of data from steroid-dependent patients with UC treated with FMT from September 2015 to September 2017 at the Dayanand Medical College, a tertiary care center in India. Fecal samples from random unrelated donors were administered through colonoscopy at weeks $0,2,6,10,14,18$, and 22. The primary outcome was achievement of steroid-free clinical remission, and the secondary outcomes were clinical response and endoscopic remission at 24 weeks. Modified intention-to-treat analysis was performed, which included subjects who underwent at least 1 FMT. Results: Of 345 patients with UC treated during the study period, 49 (14.2\%) had steroid-dependent UC. Of these 49 patients, 41 underwent FMT: 33 completed 7 sessions over 22 weeks according to the protocol, and 8 discontinued treatment (non-response, 5; lost to follow-up, 2; and fear of adverse effects, 1). At week 24, steroid-free clinical remission was achieved in 19 out of $41(46.3 \%)$ patients, whereas clinical response and endoscopic remission were achieved in 31 out of 41 (75.6\%) and 26 out of 41 (63.4\%) patients, respectively. All patients with clinical response were able to withdraw steroids. There were no serious adverse events necessitating discontinuation. Conclusions: A multisession FMT via the colonoscopic route is a promising therapeutic option for patients with steroid-dependent UC, as it can induce clinical remission and aid in steroid withdrawal. (Intest Res 2019;17:78-86)

Key Words: Fecal microbiota transplantation; Colitis, ulcerative; Real world analysis

\section{INTRODUCTION}

Corticosteroids have a significant impact in induction of remission in active UC, ${ }^{1}$ but a substantial proportion of patients may become steroid-dependent and experience adverse effects due to long-term use. These patients are managed with

Received June 12, 2018. Revised August 26, 2018.

Accepted August 30, 2018

Correspondence to Ajit Sood, Department of Gastroenterology, Dayanand Medical College \& Hospital, Tagore Nagar, Ludhiana 141001, India. Tel: +91981-5400718, Fax: +91-161-2302620, E-mail: ajitsood10@gmail.com

ORCID Ajit Sood (https://orcid.org/0000-0001-6961-6389) either immunosuppressive agents or biologics, the use of which is restricted by their limited efficacy and high cost. ${ }^{2-7}$ Fecal microbiota transplantation (FMT) has emerged as a novel approach in altering the gut microbiome and reducing colonic inflammation. This is supported by data favoring efficacy of FMT in treating recurrent Clostridium difficile infection. ${ }^{8}$ The role of FMT in active UC has been evaluated by several case reports and case series, ${ }^{9-15}$ and subsequently 4 randomized control trials (RCTs) have been published. ${ }^{16-19}$ However, these studies have significant heterogeneity with different protocols and routes of administration, doses and patient selection criteria. Furthermore, though proven to be beneficial in active 
UC, there is limited data on the efficacy and safety of FMT in patients with steroid dependent UC. ${ }^{15}$ We hereby report our real life experience with FMT in this difficult to treat group of steroid dependent UC patients.

\section{METHODS}

\section{Study Design}

This study was a single center, prospective analysis of data from patients with steroid dependent UC, who were treated with FMT at Dayanand Medical College \& Hospital, India from September 2015 through September 2017. The study was approved by the Institutional Review Board (IEC No. 2015-113). Written informed consent was obtained from each participant.

\section{Study Population}

The participants enrolled in this study described in Fig. 1. Patients with steroid dependent (defined as inability to reduce steroids below prednisolone $10 \mathrm{mg}$ /day within 3 months of starting steroids or relapse within 3 months of stopping steroids) active UC (total Mayo score varying from 4 to 10 and Mayo endoscopic sub score of $>1$ ) aged 18 to 75 years were offered FMT. The standard of care treatment including oral 5 -aminosalicylates (stable dose for 4 weeks), azathioprine (on therapy for $>3$ months and stable dose for 4 weeks), and corticosteroids were continued. These drugs were continued at the same dose, except corticosteroids, which were tapered off 2.5 mg every week starting from first session of FMT and withdrawn over next 6 to 12 weeks. Patients with previous exposure to biologics were also included. Patients who had indeterminate/CD, major comorbidities, history of colorectal malignancy, associated IBS, pregnancy, past history of surgery, exposure to antibiotics or probiotics in last 4 weeks and evi-

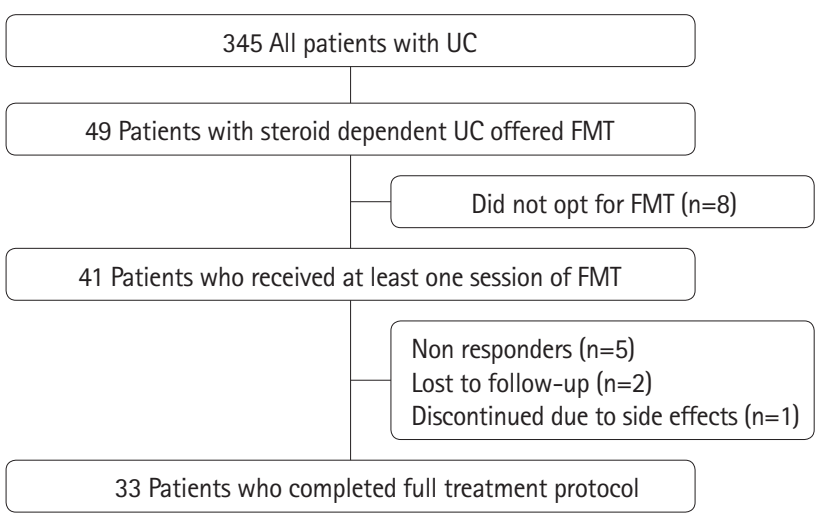

Fig. 1. Patient flow diagram. FMT, fecal microbiota transplantation. dence of infections like C. difficile, cytomegalovirus, parasites or extra-intestinal infections requiring antibiotics were not considered for FMT. Patients who were unable to hold the instilled stool sample for more than 2 hours and those with very severe UC (Mayo score, 11 and 12) where full length colonoscopy was unsafe were also not considered.

Patient demographics, disease duration, severity (defined by Mayo score), extent (Montreal classification) and treatment details were recorded. ${ }^{20}$ Clinical, laboratory parameters and colonoscopy findings were recorded at baseline and then at every subsequent FMT session.

\section{Donors}

Two healthy, asymptomatic, voluntary individuals who had no comorbidities or disorders known to be associated with changes in gut microbiota, were chosen as donors. Donor stools were screened for enteric pathogens including parasites (Entamoeba histolytica, Giardia) and bacteria (Salmonella, Shigella, Escherichia coli, Campylobacter, Yersinia, and C. difficile). The donors were accepted only if HAV IgM, HBsAg, anti-HCV antibodies, anti-human immunodeficiency virus antibodies, IgM antibodies against cytomegalovirus and tests for syphilis were negative. Prospective screening for enteric infections was done every 4 weeks and any symptoms of infection between the last screening and time of donation were enquired. The stool sample was not accepted if donors had taken antibiotics or probiotics in previous 3 months.

\section{FMT Sample Preparation, Administration and Dosing}

A freshly collected sample was inspected visually and formed stools (Bristol stool scale, 3 and 4) with no obvious blood or mucous were accepted. This sample was diluted with preservative free normal saline and homogenized using a blender (Stomacher ${ }^{\circledR} 400$ circulator; Seward Ltd, Worthing, UK) till it reached a liquid consistency. Universal precautions were observed and separate disposable bags were used for each patient. The particulate matter was filtered out, the stool slurry was filled in four $50 \mathrm{~mL}$ syringes and infused via a colonoscope in terminal ileum and cecum within 6 hours of collection of stool and 1 hour of preparation of the slurry. The recipients received samples randomly from the 2 donors (A and B), alternatively for each session (A.B.A.B.A.B.A). FMT sessions were scheduled at weeks $0,2,6,10,14,18$, and 22 . The recipients were encouraged to retain stool slurry for as long as possible and observed for 6 hours to assess stool retention time and post-procedure adverse events. 


\section{Clinical Outcomes}

At every visit, disease activity and response to therapy were assessed (using Mayo score) ${ }^{21}$ and endoscopic findings were recorded. The primary end point was achievement of steroidfree clinical remission (Mayo score $\leq 2$, with each sub-score $\leq 1$ ) at week 24 . Secondary end points were clinical response (reduction of Mayo score $\geq 30 \%$ and $\geq 3$ points compared to baseline), and endoscopic remission (Mayo score 0 or 1) at week 24 .

\section{Adverse Event Recording}

Adverse events like fever, abdominal pain, nausea, vomiting, loss of appetite, worsening of diarrhea, abdominal distension, perianal or rectal pain, flatulence, borborygmi, bloating, constipation, urinary tract infection, respiratory tract infection were recorded after each FMT session. A serious adverse event was defined as any untoward medical occurrence after FMT, resulting in inpatient hospitalization or prolongation of hospitalization, was life-threatening or resulted in death of the patient. All patients were advised to inform about any post-FMT adverse events either telephonically or by visiting outpatient department.

\section{Statistical Analysis}

Data were described in terms of range; mean $\pm \mathrm{SD}$, median, frequencies (number of cases) and relative frequencies (percentages) as appropriate. Analysis were undertaken using the modified intention-to-treat (ITT) population, which included all patients who underwent at least 1 session of FMT and repeated using the treated patient population who completed 7 sessions of FMT (per protocol analysis). Patients who did not respond to FMT, breached the study protocol or stopped FMT due to any reason were deemed as treatment failures. All statistical calculations were done using SPSS version 21.0 (IBM Corp., Armonk, NY, USA) for Microsoft Windows.

\section{RESULTS}

A total of 345 patients with UC were treated at Dayanand Medical College and Hospital, Ludhiana, India between September 2015 and September 2017. Of these, 49 (14.2\%) were steroid dependent and 30 out of 49 (61.2\%) were on immunomodulators (azathioprine) in addition to 5-aminosalicylates and corticosteroids. None of the patients was maintained on biologics due to high costs. Forty-one of these patients (mean age $36.51 \pm 10.65$ years and 24 males [58.54\%]) with steroid dependent active UC were treated with FMT (Table 1, Fig. 1). The mean Mayo score at baseline was $8.78 \pm 2.55$ and mean duration of disease was $4.59 \pm 4.23$ years. Twenty-two patients (53.65\%) were on azathioprine at time of starting FMT and 12 patients $(29.27 \%)$ had received biologics (infliximab in 7 patients and adalimumab biosimilar in 5 patients) as induction therapy in past. Eight of the 49 steroid dependent active UC patients (16.3\%) did not opt for FMT. A majority of them $(n=6$, $75 \%$ ) had apprehensions about the safety of the procedure. All these patients were maintained on azathioprine. Two healthy donors (A and B) aged 24 and 30 years respectively donated fresh fecal samples on the day of FMT. A stool bank storing the fecal samples at $-80^{\circ} \mathrm{C}$ was made to provide a backup in case of non-availability of donor, however all the FMT procedures for patients in present study were done using fresh fecal samples.

\section{Clinical Outcomes}

On a modified ITT analysis, the primary outcome (clinical remission at week 24) was achieved in 19 out of 41 patients (46.3\%) who received at least 1 session of FMT. Thirty-one patients (75.6\%) had clinical response and all of these could withdraw steroids. Endoscopic remission was noted in 26 out of 41 patients $(63.4 \%)$.

Table 1. Baseline Characteristics of Patients

\begin{tabular}{lc}
\hline Characteristic & Case $(\mathrm{n}=41)$ \\
\hline Age $(\mathrm{yr})$ & $36.5 \pm 10.7$ \\
Male sex & $24(58.5)$ \\
Disease duration (yr) & $4.6 \pm 4.2$ \\
Mayo score & $8.8 \pm 2.6$ \\
Disease extent & \\
E1 & $7(17.1)$ \\
E2 & $17(41.5)$ \\
E3 & $17(41.5)$ \\
Disease severity & \\
Mild & $4(9.8)$ \\
Moderate & $37(90.3)$ \\
Concomitant medication & \\
Mesalamine & $41(100)$ \\
Corticosteroids & $41(100)$ \\
Immunosuppressants (AZA) & $22(53.7)$ \\
Previous exposure to biological & $12(29.3)^{\mathrm{a}}$ \\
\hline
\end{tabular}

Values are presented as mean \pm SD or number (\%). ${ }^{a}$ Infliximab $(n=7)$ and adalimumab biosimilar $(n=5)$. AZA, azathioprine. 
Thirty-three patients (80.5\%) completed 7 cycles of FMT as per protocol (Table 2, Fig. 2). On a per protocol analysis, clinical remission was noted in 19 out of 33 (57.6\%), while clinical response and endoscopic remission were noted in 31 (93.9\%) and 26 patients (78.8\%), respectively. As seen in Fig. 2, the Mayo score gradually reduced after every session of FMT (Fig. 2A) and clinical response increased from $42.3 \%$ at week 2 to $93.9 \%$ at week 22 (Fig. 2B). With each procedure, the acceptability increased and patients were able to retain fecal slurry for a longer period of time (Fig. 2C).

Various factors determining the treatment outcomes were assessed (Supplementary Table 1). Patients aged $<40$ years, females, those treated early in the disease course or when the disease was mild showed better rates of clinical remission, but none of these were statistically significant. The treatment outcomes in the patients who were on immunomodulators (azathioprine) were also comparable to those treated without azathioprine.

Table 2. Clinical Outcomes in Patients Who Completed 7 Cycles of FMT ( $n=33)$

\begin{tabular}{|c|c|c|c|c|c|c|c|}
\hline Timing of FMT & Week 0 & Week 2 & Week 6 & Week 10 & Week 14 & Week 18 & Week 22 \\
\hline Mayo score & $8.9 \pm 2.5$ & $7.7 \pm 1.9$ & $6.4 \pm 2.3$ & $5.7 \pm 1.9$ & $4.8 \pm 1.9$ & $4.4 \pm 1.9$ & $3.1 \pm 1.7$ \\
\hline Clinical remission & - & 0 & $3(9.1)$ & $6(18.2)$ & 7 (21.2) & $10(30.3)$ & 19 (57.6) \\
\hline Clinical response & - & $11(42.3)$ & $17(51.5)$ & $22(66.7)$ & $28(84.8)$ & $29(87.9)$ & $31(93.9)$ \\
\hline Endoscopic remission ${ }^{\text {a }}$ & - & $4(15.3)$ & $9(27.3)$ & $12(36.4)$ & $17(51.5)$ & $21(63.4)$ & $26(78.8)$ \\
\hline Steroids successfully withdrawn & - & $1(3.9)$ & $2(6.1)$ & $15(45.5)$ & $28(84.8)$ & $29(87.9)$ & 31 (93.9) \\
\hline Slurry retention time (hr) & $2.9 \pm 1.7$ & $3.9 \pm 2.3$ & $4.4 \pm 3.6$ & $4.0 \pm 1.6$ & $4.9 \pm 3.3$ & $5.3 \pm 3.1$ & $5.2 \pm 2.8$ \\
\hline
\end{tabular}

Values are presented as mean \pm SD or number (\%).

${ }^{a}$ Endoscopic remission was defined as Mayo endoscopic score 0 or 1 . Of these patients, 0, 1, 2, 3, 3, 5, 7 patients had Mayo endoscopic score 0 at visits 1,2 , $3,4,5,6,7$ respectively.

FMT, fecal microbiota transplantation.
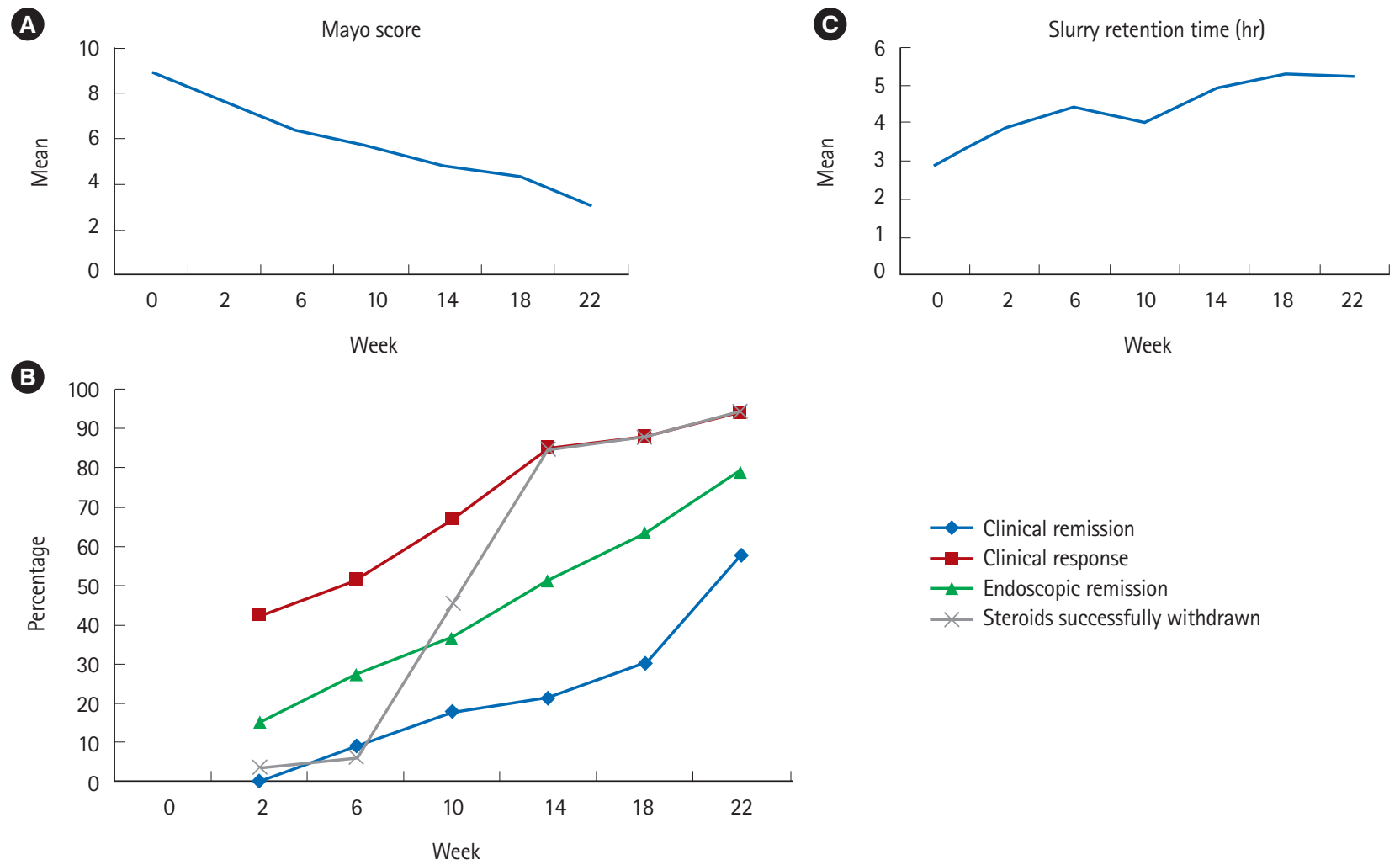

Fig. 2. Clinical outcomes in patients who completed 7 cycles of FMT ( $n=33)$. (A) Line diagram showing trend of Mayo score. (B) Line diagrams showing primary and secondary outcomes. (C) Line diagram showing trend of slurry retention time. 
Ten patients had treatment failure (non-response, 7; consent withdrawn, 1; lost to follow-up, 2). Among 7 non-responders, 2 completed 7 sessions, while 5 discontinued therapy. Two of the non-responders switched over to infliximab, 2 (previous biologic failures) underwent colectomy and 4 required increase in the dose of steroids. Only 1 patient discontinued FMT due to minor adverse events (post-procedure distension and mild pain) and she was managed with mesalamine, steroids and azathioprine.

\section{Adverse Events}

With FMT, there were no major adverse events requiring discontinuation of treatment or hospitalization though 1 patient discontinued therapy after minor side effects in the first session. Abdominal discomfort and distension were the most commonly experienced adverse events but these were transient and improved over few hours either spontaneously or with symptomatic therapy. Ten patients had worsening of diarrhea, which was self-limiting and managed with oral rehydration solution. Antibiotics were avoided as they could affect donor microbiota. With every session of FMT, the tolerability improved and number of adverse events experienced by patients reduced (Supplementary Table 2).

\section{DISCUSSION}

Our study findings suggest that 4-weekly multi-session FMT is effective for patients with steroid dependent UC in a real world setting as $75 \%$ had clinical response and could withdraw steroids. Clinical remission was achieved in $46.3 \%$ patients (19/41) and $63.4 \%(26 / 41)$ achieved endoscopic remission at week 24. The acceptability of the procedure increased with every session of FMT, with fewer adverse events and better retention times of fecal slurry.

Four high quality RCTs suggesting the efficacy of FMT in active UC patients have been published from developing countries (Table 3) ${ }^{16-19}$ and a recent systematic review and metaanalysis of these studies with 277 participants found that FMT was associated with higher rates of clinical and endoscopic remission compared with placebo. ${ }^{22}$ Despite this documented benefit, there is a significant heterogeneity in these studies, with different protocols, routes of administration, doses and patient selection criteria. ${ }^{23}$ Furthermore, only 2 of these RCTs have reported patients on steroids. ${ }^{16-19}$ Results of patients with steroid dependent UC have been reported only by Paramsothy et al., ${ }^{19}$ and though none of their 20 patients on steroids at

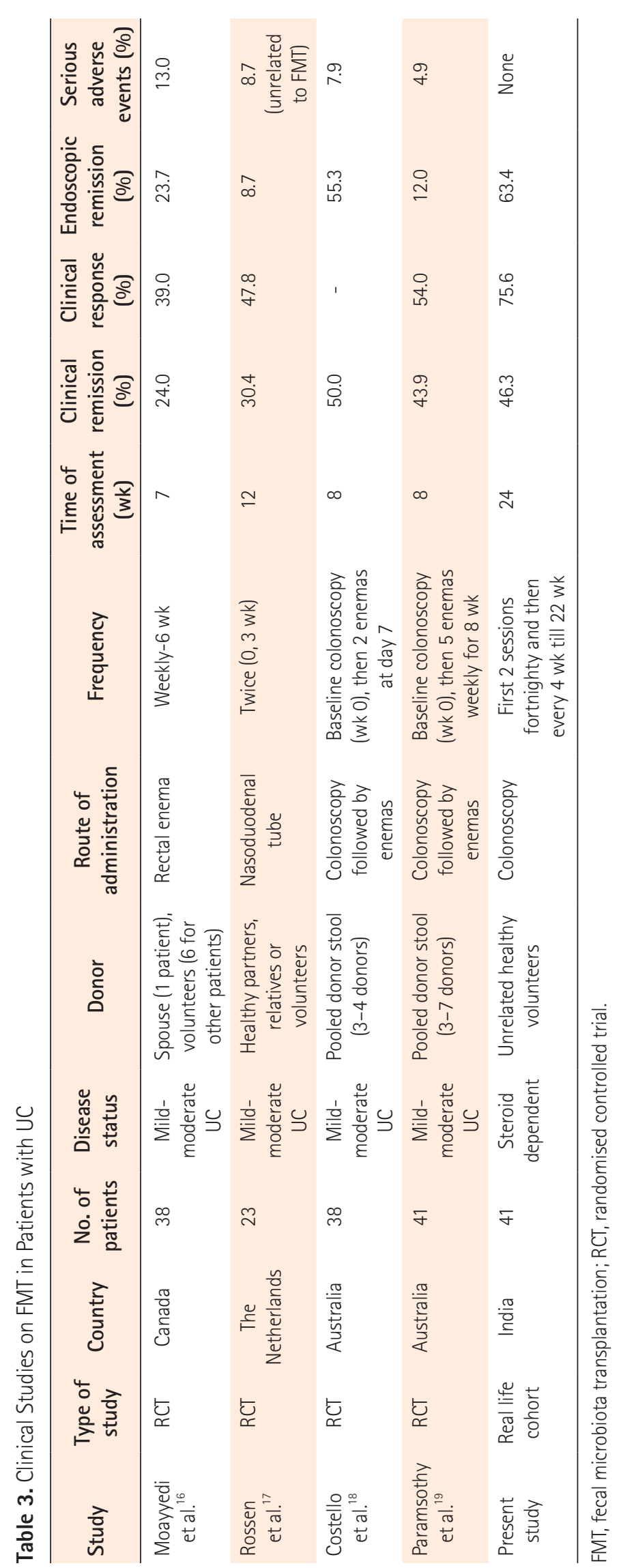


time of enrolment achieved the primary end point (steroidfree clinical remission and endoscopic remission or response at week 8) at end of masked treatment, 1 patient met the primary outcome in open label FMT. To the best of our knowledge, only 1 study has assessed response of FMT in patients with steroid dependent UC. ${ }^{15}$ In this study, steroids were withdrawn for a week before subjecting them to 2 sessions of FMT, 1 week apart. After this, steroids were re-introduced in a short course of 2 to 4 weeks. Clinical improvement was seen in 8 out of 14 (57\%) patients, among which $5(35.7 \%)$ received 1 FMT therapy, 1 (7.1\%) received 2 FMTs, and 2 (14.2\%) received 2 FMTs plus a scheduled course of steroids. Patients in our study on the other hand, were recruited while being on steroids and addition of FMT by a multi-session protocol resulted in clinical response in $75 \%$ patients, all of whom could withdraw steroids.

We attribute better response rates in our study to (a) the use of a multi-session protocol over 22 weeks and (b) colonoscopic route of administration. The optimum intensity and duration of FMT has not been defined by any study so far. A single session of FMT has been shown to be efficacious in patients with $C$. difficile infection, where antibiotics disrupt the indigenous gut microbiota. ${ }^{24}$ However, pathogenesis of UC involves complex pathologic mechanisms including immunologic, dysbiosis and genetic factors ${ }^{25}$ and the therapeutic microbial manipulation may require multiple sessions. The reported frequency of administration of FMT varies from limited dosing schedules of 1 to 2 doses to extremely intense schedule of 41 doses. ${ }^{16-19}$ Though our protocol was not very intense like that of Paramsothy et al., ${ }^{19}$ it was spread over a longer period (22 weeks) and yielded similar results (steroid-free remission in 46.3\%). It must be highlighted that we used FMT in a more difficult to treat group of steroid dependent UC patients (as compared to all active UC patients reported earlier). Clinical response was seen in $75.6 \%$ patients (31/41), of which $90.3 \%$ (28/31) responded by week 14 and the response rates further improved till completion of study period. Once achieved, the response was sustained in these patients till the end of study period, that is 24 weeks.

The colonoscopic route of administration may have resulted in better outcomes in our study. Instillation of the fecal slurry in the upper GI tract may not be effective as it has been hypothesized that gastric acid can destroy Bacteroides and Firmicutes. ${ }^{26}$ However, studies delivering fecal slurry via upper GI tract have either administered proton pump inhibitors or delivered the material into duodenum or jejunum to negate the effect of gastric acid. The RCTs with a colonic or rectal instillation of fecal slurry ${ }^{18,19}$ have shown better response compared to the oral route of administration. ${ }^{17}$ In the most recent RCT by Paramsothy et al., ${ }^{19}$ patients received first infusion colonoscopically and then 40 rectal enemas in 8 weeks. These infusions were dispensed fortnightly and stored in a home freezer at $-20^{\circ} \mathrm{C}$ before daily administration. However, in a real life setting in a developing country like India, storage of fecal matter in a home refrigerator with other food articles is not acceptable and maintaining the desired temperature of $-20^{\circ} \mathrm{C}$ may not be feasible. Furthermore, ensuring proper administration, retention and compliance may not be feasible with self-administered home enemas. We chose colonoscopic route of instillation, as the therapy was directly observed by the practitioner and larger volumes could be administered with better slurry retention times. Unlike administration into upper GI tract, there was no risk of aspiration and the microbial uptake may have been better, as inflammation in UC starts in rectum and proceeds proximally, and dysbiosis is expected to be more in inflamed areas than the non-inflamed ones. ${ }^{27}$

The role of pre-FMT bowel preparation is yet unclear. While a few studies favor bowel lavage to help successful colonization of the donor microbiota in the recipient by diminishing the host microbiome, studies where only retention enemas have yielded positive results suggest that it may not be needed ${ }^{18,19,28}$ We used adequate bowel preparation before each visit which helped in providing a proper mucosal assessment during each visit, in addition to the probable benefit on change in microbiome.

Another factor which may determine the efficacy of FMT is the donor. The random use of 2 unrelated donors in our study may have resulted in better outcomes in our study as related donors may have similar microbiome as the recipients due to common genetic and environmental factors. ${ }^{9,13,15}$ Also, the use of multiple donors might have increased the microbial heterogeneity, thus resulting in better outcomes, as shown in other studies. $^{19,29}$

Patients in our study had positive attitudes towards FMT, though a few had initial concerns regarding repeated procedures, especially with bowel preparation. Except 1 patient who withdrew after the first session, all patients were willing to continue FMT due to apparent clinical improvement and ability to withdraw steroids. There were no major adverse events and the tolerability and slurry retention times were noted to increase with each session of FMT. Similar positive attitudes of patients towards FMT have been reported from other parts of the world. ${ }^{30,31}$ The cost of FMT at our center was approximate- 
ly 50 US dollars, which was much cheaper than maintenance with biologics and immunomodulators.

Eight patients with steroid dependent UC who did not opt for FMT were maintained on azathioprine for 24 weeks and among these, steroid-free clinical remission was noted in 2 (25\%) and clinical response in 4 patients (50\%). The use of biologics was limited in our center due to high cost. However, the number of patients who could discontinue steroids on infliximab in the ACT 1 and ACT 2 trials ( $21.7 \%$ and $22.8 \%$ respectively at week 30 , and $21 \%$ at week 54 in ACT 1) was much lower than our patients treated with FMT. ${ }^{5}$ In the UC-SUCCESS trial, steroid-free remission at week 16 was $22.1 \%$ with infliximab, 23.7\% with azathioprine and 39.7\% with the combination. ${ }^{6}$ FMT in patients with steroid dependent UC was thus more efficacious than both azathioprine and infliximab.

Our study had a few limitations. First, being a real-world analysis, there was no control arm to compare the efficacy of FMT. Second, we could not analyze the gut microbiome of the patients and donors due to financial limitations. The previous studies that have profiled the microbiota in IBD patients have reported reduction in phyla Bacteroidetes and Firmicutes and increase in Proteobacteria. ${ }^{32-34}$ FMT results in an increase in the microbial diversity and post FMT, the microbiome has been reported to resemble healthy controls. ${ }^{16-19}$ However, despite extensive ongoing research in this field, specific gut bacterial species which result in development of IBD are still unclear. The high rate of steroid-free response and clinical remission achieved in the difficult to treat steroid dependent UC patients noted in our study suggests the need of larger studies with detailed microbiome analysis to elucidate the possible microbiome changes in this group of patients.

In conclusions, our study suggests that a multi-session colonoscopic FMT is a promising strategy for patients with steroid dependent UC, as it improves rates of steroid-free clinical remission, clinical response (and withdrawal of steroids) and endoscopic remission.

\section{FINANCIAL SUPPORT}

The authors received no financial support for the research, authorship, and/or publication of this article.

\section{CONFLICT OF INTEREST}

No potential conflict of interest relevant to this article was reported.

\section{AUTHOR CONTRIBUTION}

All authors have contributed to and agreed on the content of the manuscript, and the respective roles of each author are listed below:

Sood A and Mahajan R: conception and design; collection, analysis and interpretation of the data; drafting of the article; critical revision of the article for important intellectual content; final approval of the article. Juyal G: analysis and interpretation of the data; drafting of the article; critical revision of the article for important intellectual content; final approval of the article. Midha V: conception and design; analysis and interpretation of the data; drafting of the article; critical revision of the article for important intellectual content; final approval of the article. Grewal CS: collection, analysis and interpretation of the data, critical revision of the article for important intellectual content; final approval of the article. Mehta V: analysis and interpretation of the data, critical revision of the article for important intellectual content; final approval of the article. Singh A: collection, analysis and interpretation of the data; drafting of the article; critical revision of the article for important intellectual content; final approval of the article. Joshi MC: drafting of the article; critical revision of the article for important intellectual content; final approval of the article. Narang V, Kaur K, and Sidhu H: data analysis, critical revision of the article for important intellectual content; final approval of the article.

\section{REFERENCES}

1. Ekbom A, Helmick CG, Zack M, Holmberg L, Adami HO. Survival and causes of death in patients with inflammatory bowel disease: a population-based study. Gastroenterology 1992;103: 954-960.

2. Fraser AG, Orchard TR, Jewell DP. The efficacy of azathioprine for the treatment of inflammatory bowel disease: a 30 year review. Gut 2002;50:485-489.

3. Ardizzone S, Maconi G, Russo A, Imbesi V, Colombo E, Bianchi Porro G. Randomised controlled trial of azathioprine and 5 -aminosalicylic acid for treatment of steroid dependent ulcerative colitis. Gut 2006;55:47-53.

4. Adler DJ, Korelitz BI. The therapeutic efficacy of 6-mercaptopurine in refractory ulcerative colitis. Am J Gastroenterol 1990; 85:717-722.

5. Rutgeerts P, Sandborn WJ, Feagan BG, et al. Infliximab for induction and maintenance therapy for ulcerative colitis. N Engl J Med 2005;353:2462-2476. 
6. Panaccione R, Ghosh S, Middleton S, et al. Combination therapy with infliximab and azathioprine is superior to monotherapy with either agent in ulcerative colitis. Gastroenterology 2014;146:392-400.

7. Armuzzi A, Pugliese D, Danese S, et al. Infliximab in steroiddependent ulcerative colitis: effectiveness and predictors of clinical and endoscopic remission. Inflamm Bowel Dis 2013; 19:1065-1072.

8. Girotra M, Garg S, Anand R, Song Y, Dutta SK. Fecal microbiota transplantation for recurrent Clostridium difficile infection in the elderly: long-term outcomes and microbiota changes. Dig Dis Sci 2016;61:3007-3015.

9. Ren R, Sun G, Yang Y, et al. A pilot study of treating ulcerative colitis with fecal microbiota transplantation. Zhonghua Nei Ke Za Zhi 2015;54:411-415.

10. Colman RJ, Rubin DT. Fecal microbiota transplantation as therapy for inflammatory bowel disease: a systematic review and meta-analysis. J Crohns Colitis 2014;8:1569-1581.

11. Uygun A, Ozturk K, Demirci H, et al. Fecal microbiota transplantation is a rescue treatment modality for refractory ulcerative colitis. Medicine (Baltimore) 2017;96:e6479.

12. Kunde S, Pham A, Bonczyk S, et al. Safety, tolerability, and clinical response after fecal transplantation in children and young adults with ulcerative colitis. J Pediatr Gastroenterol Nutr 2013; 56:597-601.

13. Suskind DL, Singh N, Nielson H, Wahbeh G. Fecal microbial transplant via nasogastric tube for active pediatric ulcerative colitis. J Pediatr Gastroenterol Nutr 2015;60:27-29.

14. Angelberger S, Reinisch W, Makristathis A, et al. Temporal bacterial community dynamics vary among ulcerative colitis patients after fecal microbiota transplantation. Am J Gastroenterol 2013;108:1620-1630.

15. Cui B, Li P, Xu L, et al. Step-up fecal microbiota transplantation strategy: a pilot study for steroid-dependent ulcerative colitis. J Transl Med 2015;13:298.

16. Moayyedi P, Surette MG, Kim PT, et al. Fecal microbiota transplantation induces remission in patients with active ulcerative colitis in a randomized controlled trial. Gastroenterology 2015; 149:102-109.

17. Rossen NG, Fuentes S, van der Spek MJ, et al. Findings from a randomized controlled trial of fecal transplantation for patients with ulcerative colitis. Gastroenterology 2015;149:110-118.

18. Costello SP, Waters O, Bryant RV, et al. Short duration, low intensity, pooled fecal microbiota transplantation induces remission in patients with mild-moderately active ulcerative colitis: a randomised controlled trial. Gastroenterology 2017;

\section{2:S198-S199.}

19. Paramsothy S, Kamm MA, Kaakoush NO, et al. Multidonor intensive faecal microbiota transplantation for active ulcerative colitis: a randomised placebo-controlled trial. Lancet 2017; 389:1218-1228.

20. Satsangi J, Silverberg MS, Vermeire S, Colombel JF. The Montreal classification of inflammatory bowel disease: controversies, consensus, and implications. Gut 2006;55:749-753.

21. Lewis JD, Chuai S, Nessel L, Lichtenstein GR, Aberra FN, Ellenberg JH. Use of the noninvasive components of the Mayo score to assess clinical response in ulcerative colitis. Inflamm Bowel Dis 2008;14:1660-1666.

22. Narula N, Kassam Z, Yuan Y, et al. Systematic review and meta-analysis: fecal microbiota transplantation for treatment of active ulcerative colitis. Inflamm Bowel Dis 2017;23:1702-1709.

23. Fairhurst NG, Travis SP. Why is it so difficult to evaluate faecal microbiota transplantation as a treatment for ulcerative colitis? Intest Res 2018;16:209-215.

24. Britton RA, Young VB. Role of the intestinal microbiota in resistance to colonization by Clostridium difficile. Gastroenterology 2014;146:1547-1553.

25. Serban DE. Microbiota in inflammatory bowel disease pathogenesis and therapy: is it all about diet? Nutr Clin Pract 2015; 30:760-779.

26. Damman CJ, Miller SI, Surawicz CM, Zisman TL. The microbiome and inflammatory bowel disease: is there a therapeutic role for fecal microbiota transplantation? Am J Gastroenterol 2012;107:1452-1459.

27. Walker AW, Sanderson JD, Churcher C, et al. High-throughput clone library analysis of the mucosa-associated microbiota reveals dysbiosis and differences between inflamed and noninflamed regions of the intestine in inflammatory bowel disease. BMC Microbiol 2011;11:7.

28. Agrawal M, Aroniadis OC, Brandt LJ, et al. The long-term efficacy and safety of fecal microbiota transplant for recurrent, severe, and complicated Clostridium difficile infection in 146 elderly individuals. J Clin Gastroenterol 2016;50:403-407.

29. Vermeire S, Joossens M, Verbeke K, et al. Donor species richness determines faecal microbiota transplantation success in inflammatory bowel disease. J Crohns Colitis 2016;10:387-394.

30. Xu L, Zhang T, Cui B, et al. Clinical efficacy maintains patients' positive attitudes toward fecal microbiota transplantation. Medicine (Baltimore) 2016;95:e4055.

31. Damman CJ, Brittnacher MJ, Westerhoff M, et al. Low level engraftment and improvement following a single colonoscopic administration of fecal microbiota to patients with ulcer- 
ative colitis. PLoS One 2015;10:e0133925.

32. Peterson DA, Frank DN, Pace NR, Gordon JI. Metagenomic approaches for defining the pathogenesis of inflammatory bowel diseases. Cell Host Microbe 2008;3:417-427.

33. Manichanh C, Rigottier-Gois L, Bonnaud E, et al. Reduced diversity of faecal microbiota in Crohn's disease revealed by a metagenomic approach. Gut 2006;55:205-211.

34. Gophna U, Sommerfeld K, Gophna S, Doolittle WF, Veldhuyzen van Zanten SJ. Differences between tissue-associated intestinal microfloras of patients with Crohn's disease and ulcerative colitis. J Clin Microbiol 2006;44:4136-4141. 


\section{See "Efficacy of fecal microbiota therapy in steroid dependent ulcerative colitis: a real world intention-to-treat analy- sis" on page 78-86.}

Supplementary Table 1. Factors Determining Outcomes with FMT in Patients with Steroid Dependent UC

\begin{tabular}{|c|c|c|c|c|c|c|c|}
\hline Factor & $\begin{array}{c}\text { Total no. of patients } \\
\text { who received FMT } \\
(n=41)\end{array}$ & $\begin{array}{l}\text { Clinical } \\
\text { remission } \\
(n=19)\end{array}$ & $P$-value & $\begin{array}{l}\text { Clinical } \\
\text { response } \\
(n=31)\end{array}$ & $P$-value & $\begin{array}{l}\text { Endoscopic } \\
\text { remission } \\
(n=26)\end{array}$ & $P$-value \\
\hline Age (yr) & & & 0.73 & & 0.12 & & 0.06 \\
\hline$\leq 40$ & 29 & $14(48.3)$ & & 20 (69.0) & & $21(72.4)$ & \\
\hline$>41$ & 12 & $5(41.7)$ & & $11(91.7)$ & & $5(41.7)$ & \\
\hline Sex & & & 0.48 & & 0.40 & & 0.60 \\
\hline Male & 24 & $10(41.7)$ & & $17(70.8)$ & & $16(66.7)$ & \\
\hline Female & 17 & 9 (52.9) & & 14 (82.4) & & $10(58.8)$ & \\
\hline Disease duration (yr) & & & 0.18 & & 0.57 & & 0.88 \\
\hline$\leq 2$ & 17 & 10 (58.8) & & 12 (70.6) & & $11(64.7)$ & \\
\hline$>2$ & 24 & $9(37.5)$ & & $19(79.2)$ & & $15(62.5)$ & \\
\hline Disease severity & & & 0.23 & & 0.97 & & 0.61 \\
\hline Mild & 4 & $3(75.0)$ & & $3(75.0)$ & & $3(75.0)$ & \\
\hline Moderate & 37 & 16 (43.2) & & $28(75.7)$ & & $23(62.2)$ & \\
\hline AZA & & & 0.90 & & 0.60 & & 0.50 \\
\hline $\mathrm{FMT}+\mathrm{AZA}$ & 22 & $10(45.5)$ & & $17(54.8)$ & & $15(68.2)$ & \\
\hline FMT alone & 19 & $9(47.4)$ & & $14(45.2)$ & & $11(57.9)$ & \\
\hline
\end{tabular}

Values are presented as number (\%).

FMT, fecal microbiota transplantation; AZA, azathioprine.

Supplementary Table 2. Adverse Events Noted with Fecal Microbiota Transplantation

\begin{tabular}{|c|c|c|c|c|c|c|c|}
\hline \multirow[b]{2}{*}{ Adverse event } & \multicolumn{7}{|c|}{ No. of patients affected } \\
\hline & $\begin{array}{l}\text { Week } 0 \\
(n=41)\end{array}$ & $\begin{array}{l}\text { Week } 2 \\
(n=40)\end{array}$ & $\begin{array}{l}\text { Week } 6 \\
(n=38)\end{array}$ & $\begin{array}{c}\text { Week } 10 \\
(n=36)\end{array}$ & $\begin{array}{c}\text { Week } 14 \\
(n=36)\end{array}$ & $\begin{array}{c}\text { Week } 18 \\
(n=33)\end{array}$ & $\begin{array}{c}\text { Week } 22 \\
(n=33)\end{array}$ \\
\hline Abdominal discomfort & $26(63.4)$ & $20(50.0)$ & $10(26.3)$ & $5(13.9)$ & $4(11.1)$ & $1(3.0)$ & $2(6.1)$ \\
\hline Abdominal distension & $14(34.1)$ & $8(20.0)$ & $2(5.3)$ & $4(11.1)$ & $1(2.8)$ & 0 & 0 \\
\hline Fever & $4(9.8)$ & $4(10.0)$ & $2(5.3)$ & $2(5.6)$ & 0 & 0 & 0 \\
\hline Worsening of diarrhoea & $4(9.8)$ & $2(5.0)$ & $1(2.6)$ & $3(8.3)$ & 0 & 0 & 0 \\
\hline Rectal bleeding & 0 & 0 & 0 & $2(5.6)$ & 0 & 0 & 0 \\
\hline Flatulence & $2(4.9)$ & $2(5.0)$ & 0 & 0 & 0 & 0 & 0 \\
\hline Borborygmi & 0 & 0 & $1(2.6)$ & 0 & $1(2.8)$ & $2(6.1)$ & 0 \\
\hline Fatigue & $2(4.9)$ & 0 & $1(2.6)$ & $1(2.8)$ & $1(2.8)$ & 0 & 0 \\
\hline Total no. of events & 52 & 36 & 17 & 17 & 7 & 3 & 2 \\
\hline
\end{tabular}

